\title{
Underwater vehicle navigation using diffusion-based trajectory observers
}

\author{
Jérôme Jouffroy and Jan Opderbecke
}

\begin{abstract}
This paper addresses the issue of estimating underwater vehicle trajectories using gyro-Doppler (body-fixed velocities) and acoustic positioning signals (earth-fixed positions). The approach consists of diffusion-based observers processing a whole trajectory segment at a time, allowing the consideration of important practical problems such as different information update rates, outages, and outliers in a very simple framework. Results of contraction theory are used to prove that the observers are convergent, i.e. stable in the incremental sense. Simulation and experimental results are presented to illustrate the potential of application of the method.
\end{abstract}

\section{INTRODUCTION}

Knowing the horizontal position of an underwater vehicle precisely enough as it moves above the sea floor is of great importance. This is true not only for precise maneuvering and other control-based concerns, but also because the accurate knowledge of the vehicle trajectory is often the first step toward performing other tasks, whether these are computed online (maneuvering, docking, marking interesting locations, collecting samples, etc.) or offline (cartography, video mosaicking, etc.)[53].

The precision of position sensing is limited and depends on many different factors, like the kind and type of sensors used (Doppler-gyrocompass, Long BaseLine (LBL) and Ultra-Short BaseLine (USBL) acoustic positioning systems, Inertial Measurement Unit (IMU), Global Positioning System (GPS), etc.) as well as the events that are connected with sensors features or underwater environmental conditions like noise, sensor misalignment, outliers and outages (see [58], [57], [22]).

Indeed, most underwater vehicle navigation systems use long-range acoustic positioning like LBL or USBL which, roughly speaking, consist of interrogating transponder beacons. This information suffers from a poor precision due to a low refresh rate, an important noise, as well as a high percentage of data loss and outliers due to sound reflections, disturbances, etc. Alternatively, another available measurement is the body-fixed velocities given by a Doppler-effect sensor which, when coupled to a gyrocompass, gives the velocity of the vehicle in an earth-fixed reference frame. Though this information is not as corrupted by noise as the acoustic positioning data, the fact that a time-integration process, necessary to get a position estimation, can lead to large deviations in the presence of noise or misalignment of the gyrocompass, is not satisfactory either.

Several studies have addressed the problem of vehicle trajectory estimation by combining the abovementioned measurements, i.e. acoustic positions with velocity data [2], [57], [22]. The effect of loss of information on these methods seems to lead to non-smooth dynamic behavior of the vehicle and hence might not be suitable for cartography or video-mosaic issues for example.

This paper presents an approach for estimating the trajectory of an underwater vehicle using mainly gyroDoppler measurements (speed measurements) and an acoustic positioning system (horizontal position). The method is based on the use of diffusion-based observers which, contrary to traditional state observers, are able to process whole segments of the system trajectory at a time. Thus, these will be referred to as

Jérôme Jouffroy is with the Center for Ships and Ocean Structures (CESOS), Norwegian University of Science and Technology (NTNU), NO-7491 Trondheim, Norway (e-mail: jouffroy@marin.ntnu.no).

Jan Opderbecke is with the Underwater Robotics, Navigation and Vision Department (RNV), French Institute for Ocean Research (IFREMER), 83507 La-Seyne-sur-Mer, France (e-mail: Jan.Opderbecke@ifremer.fr). 
trajectory observers. As will be seen, one of the main advantages of such an approach is that, unlike other studies on navigation (see for example [2], [40], [1], [51], [18], [4]), many of the difficulties associated with the Doppler-gyro / USBL combination (differing information rates, Doppler-gyro drift, outliers, outages of the acoustic positioning system, etc.) are treated very simply by starting with the same basic equations, thus leading to a quite unified view of the problem at hand. Another significant advantage of the method is that the Partial Differential Equation (PDE) framework in which it is embedded allows rigorous practical and theoretical study as many mathematical tools are available to address important issues such as stability, robustness, implementation, while the diffusion metaphor used throughout the paper enables to maintain the algorithmic description in a relatively simple, intuitive and compact form.

The rest of the paper is organized as follows. After this introduction, a few elements of underwater vehicle navigation in the horizontal plane are given in section II. In section III, we introduce several diffusion-based trajectory observers, each one of them addressing a different issue, i.e. smooth combined Doppler / acoustic positioning trajectory estimation, acoustic data smoothing, "rendezvous" time constraints, gyrocompass bias estimation, and sound velocity profile estimation. These observers being primarily meant for offline purposes, we also briefly show how the approach extends to on-line versions by the addition of a simple term allowing the estimated segments of trajectory to "slide" in time. Because stability is one of the key issues in observer design, the finite element approximations that are used to implement each trajectory observers are proven to be contracting, i.e. stable in the sense of contraction analysis [26], [27], [30], [29], [28], [24] and therefore exhibit an exponential convergence. Computer simulations are also presented to demonstrate the observers behavior. Experimental results of the combined Doppler / acoustic positioning trajectory observer from operation of the deep-sea ROV Victor 6000 (see figure 1) are briefly discussed in section IV. Brief concluding remarks are given in section V, while basic results of contraction analysis are given in the appendix.

Earlier versions of some parts of this paper appeared in [20], [19].

\section{UNDERWATER VEHICLE NAVIGATION}

In the following, we consider trajectory estimation of vehicles evolving on the horizontal plane, whose kinematics will be written as follows [12]

$$
\left(\begin{array}{c}
\dot{x} \\
\dot{y}
\end{array}\right)=\left(\begin{array}{cc}
\cos \psi & -\sin \psi \\
\sin \psi & \cos \psi
\end{array}\right)\left(\begin{array}{l}
u \\
v
\end{array}\right)
$$

where the vector $(x, y)^{T}$ stands for the position of the vehicle in an earth-fixed frame, $(u, v)^{T}$ is the vector of body-fixed velocities, while $\psi$ is the heading of the vehicle that is used to compute the time-varying rotation from the body-fixed or vehicle frame to the earth-fixed one.

By using a complex setting and defining $X \triangleq x+i y$ and $V \triangleq u+i v$, where $i$ is the imaginary number, equation (1) is reduced to

$$
\dot{X}=e^{i \psi} V
$$

This complex notation will be used in the rest of the paper. A position given by the acoustic positioning system (USBL or LBL) is denoted as $X_{a c}$. As mentioned earlier on, the measurements are typically corrupted by noise and suffers from a high percentage of outages and outliers, while the update rate of the positioning information lies between 0.1 and $1 \mathrm{~Hz}$, depending on the system in use.

The body-fixed velocities are provided by a bottom-lock Doppler sonar, consisting of four downwardlooking beam transducers that measure the velocity relative to the seafloor. These velocities, that we denote here $V_{d o p}$ are then transformed into the earth-fixed coordinates using the standard rotation

$$
V_{g e o}=e^{i \psi} V_{d o p}
$$




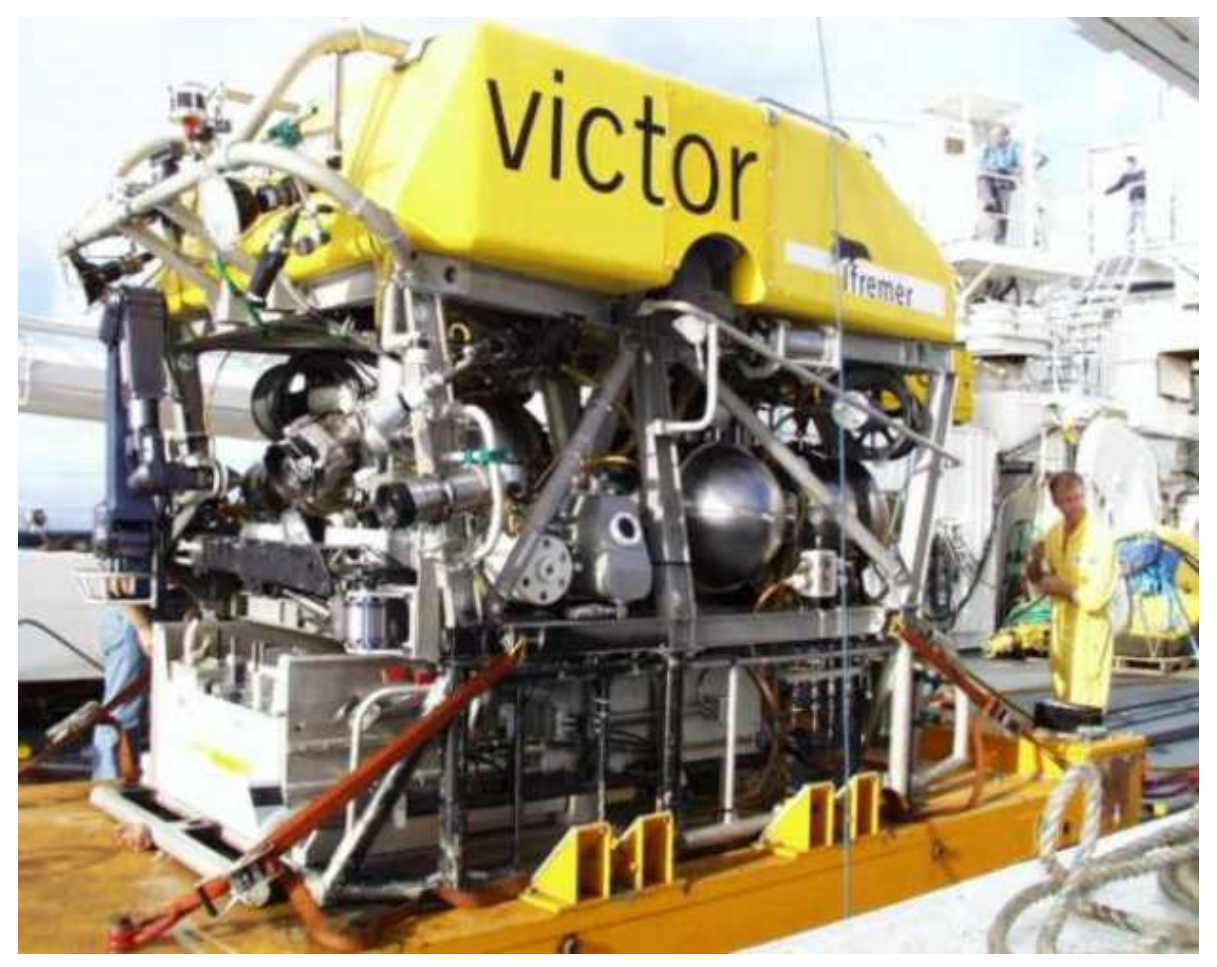

Fig. 1. IFREMER ROV Victor 6000

where the heading $\psi$ is measured by a gyrocompass, and $V_{g e o}$ is the speed vector in the earth-fixed frame. $V_{d o p}$ and $V_{g e o}$ are typically less noisy than the acoustic positioning measurements $X_{a c}$ while their update rate are higher ( $5 \mathrm{~Hz}$ for the Victor 6000 for example). When the heading is measured by an optical gyrocompass, the precision and update rates of $\psi$ are generally excellent. A dead-reckoning process, i.e. a time integration of the velocities might thus seem to be appropriate due to the good sensor performances. However, these are counterbalanced by other factors such as a misalignment of the gyrocompass, the problem of the initial position determination in the dead-reckoning, and accumulation of small noise due to the time-integration process that could lead to significant deviations of the estimated trajectory with respect to the real path of the vehicle [57].

\section{DIFFUSION-BASED TRAJECTORY OBSERVERS}

In order to differentiate trajectory observers from a usual state observer, also called Luenberger observer [34], let us recall that the state of a system represented by equation (2) and more generally by the nonlinear $\dot{X}=f(X)$ can be seen as a moving particle in the state-space, whose path is referred to as a trajectory. Using this interpretation, a state observer is nothing but a tracking system whose corresponding particle, usually denoted as $\hat{X}$, follows $X$ based on the signals that are available for measurement. Ideally, and in the absence of noise, the particle $\hat{X}$ will use the time evolution to converge towards $X$.

Such a point-of-view, the one of state observers, is of course especially suited to online purposes related with control architectures. However, it is not appropriate for offline tasks related for example to videomosaicking because of the uncertainty due to an error in the initial guess, i.e. the initial condition of the state observer is concentrated in the beginning of the trajectory, thus leading to possibly large errors in the mosaicking process. Also, when it comes to underwater navigation, the presence or the absence of an acoustic position has an important impact on the estimator design issue. Indeed, if it is required that a vehicle goes back to a position it has been to before, it might be of interest to re-estimate this position to be certain to find it, hence to re-estimate the past based on the present available information. This is especially the case in the context of acoustic positioning and of data interruption. 
Hence, the common thread to these different issues being the estimation of the path of the vehicle, define the trajectory estimate $\hat{X}(s, t)$. This estimate is a function of the trajectory time $s$, i.e. the temporal position of the vehicle along its path, with $s \in\left[s_{b}, s_{e}\right]$ in seconds. Additionally, the estimation process being also iterative (i.e. it improves with iterations), $\hat{X}$ depends on $t \in \mathbb{R}^{+}$, where $t$ represents the continuous counterpart of iterations, that will be referred to as the improvement time. Note that in the traditional state observer context, $s$ and $t$ are combined into one single variable, usually christened $t$, since the observer uses the evolution of the trajectory time in the iterative process to converge to the particle of the system under observation.

In the following, we show how a trajectory observer is constructed and present a few trajectory observers used to deal with offline underwater navigation-related problems first, and then sketch how online extensions of these can be done.

\section{A. Combined acoustic positioning / Doppler trajectory estimation}

When combining acoustic positioning measurements with Doppler velocity estimates, the most suitable trajectory estimation should take into account the different features of these data, i.e. this estimation should reproduce the measured vehicle motion while respecting global position references given by the acoustic positioning system.

Hence, introduce the following integral criterion that balances the match of $\hat{X}$ with the acoustic positioning signal $X_{a c}(s)$ and its derivative with respect to $s$ with $V_{\text {geo }}(s)$

$$
\int_{s_{b}}^{s_{e}}\left(\nabla \hat{X}-V_{g e o}\right)^{2}+k_{X}(s)\left(\hat{X}-X_{a c}\right)^{2} d s
$$

where $\nabla \hat{X} \triangleq \partial \hat{X} / \partial s . k_{X}(s)$ is a tuning function allowing to weigh the significance of the acoustic positioning measurements over the final trajectory estimation. It also allows to take into account in an explicit way the discrete-in-time feature of $X_{a c}$ by writing $k_{X}$ as

$$
k_{X}(s)=K \sum_{i=1}^{n_{s}} \delta\left(s-s_{i}\right)
$$

where $K$ is a strictly positive constant accounting for the weighting of $X_{a c}, \delta(\cdot)$ stands for the Dirac delta function, and $s_{i} \in\left[s_{b}, s_{e}\right]$ are the $n_{s}$ trajectory times for which the acoustic positioning signal is available. Note that such a framework allows to deal with a non-periodic acoustic positioning signal which appears when many outages perturb the signal. Also, the outliers are treated as outages as they are simply discarded whenever detected.

Minimizing the integral in (4) in an iterative way is performed easily by computing the gradient descent of the Euler-Lagrange equation of (4)[25], which can be obtained using the following PDE

$$
\frac{\partial \hat{X}}{\partial t}=\nabla\left(\nabla \hat{X}-V_{g e o}(s)\right)+k_{X}(s)\left(X_{a c}(s)-\hat{X}\right)
$$

This equation can be interpreted as a diffusion-reaction process whose diffusion behavior, usually merely $\nabla^{2} \hat{X}$, is here guided by the velocity measurements. The role of the reaction/source term $k_{X}\left(X_{a c}-\hat{X}\right)$ is to ensure that the final estimate $\hat{X}$ will be close to the acoustic positions through a feedback driven by $X_{a c}$.

One way to obtain a finite element implementation of (6) is to first consider the discrete approximation of the heat equation

$$
\frac{\partial \hat{X}}{\partial t}(s, t)=\nabla^{2} \hat{X}(s, t)
$$


where the Laplace operator $\nabla^{2} \hat{X}=\Delta \hat{X}$ can be approximated as

$$
\nabla^{2} \hat{\mathbf{X}}=\frac{(n+1)^{2}}{S^{2}}\left(\begin{array}{ccccc}
-2 & 1 & 0 & 0 & \ddots \\
1 & -2 & 1 & \ddots & \ddots \\
0 & 1 & \ddots & \ddots & 0 \\
0 & \ddots & \ddots & -2 & 1 \\
\ddots & \ddots & 0 & 1 & -2
\end{array}\right) \hat{\mathbf{X}}+\left(\begin{array}{c}
\hat{X}_{b} \\
0 \\
\vdots \\
0 \\
\hat{X}_{e}
\end{array}\right)
$$

where $n$ is the number of discrete elements of the trajectory segment and the dimension of the finite state vector $\hat{\mathbf{X}}$ and $S$ is the segment length in seconds. $\hat{X}_{b}$ and $\hat{X}_{e}$ are Dirichlet boundary conditions which, from a trajectory observer perspective, mean that the extremum conditions, represented by the start and end points of the vehicle path, are known.

In the case where only velocity measurements are known at the boundaries, we have $\nabla \hat{X}_{b}$ and $\nabla \hat{X}_{e}$ instead of $\hat{X}_{b}$ and $\hat{X}_{e}$. This leads to the finite state-space representation for the approximation of (7)

$$
\dot{\hat{\mathbf{X}}}=\mathbf{L}_{D} \hat{\mathbf{X}}+\mathbf{B}_{D}
$$

where the matrix $\mathbf{L}_{D}$ stands for the discrete approximation of the Laplacian operator with two homogeneous Dirichlet boundary conditions, and $\mathbf{B}_{D}$ is the vector of boundary conditions. In the case where these are Neumann instead of Dirichlet boundary conditions, replace $\mathbf{L}_{D}$ and $\mathbf{B}_{D}$ by $\mathbf{L}_{N}$ and $\mathbf{B}_{N}$ which are easily obtained by writing the discrete Laplace operator as

$$
\nabla^{2} \hat{\mathbf{X}}=\frac{(n+1)^{2}}{S^{2}}\left(\begin{array}{ccccc}
-1 & 1 & 0 & 0 & \ddots \\
1 & -2 & 1 & \ddots & \ddots \\
0 & 1 & \ddots & \ddots & 0 \\
0 & \ddots & \ddots & -2 & 1 \\
\ddots & \ddots & 0 & 1 & -1
\end{array}\right) \hat{\mathbf{X}}+\frac{S}{n+1}\left(\begin{array}{c}
-\nabla \hat{X}_{b} \\
0 \\
\vdots \\
0 \\
\nabla \hat{X}_{e}
\end{array}\right)
$$

Note that we can also have the two kinds of boundary conditions in one equation, i.e. for example a Neumann one for $s_{b}$ and a Dirichlet one for $s_{e}$. Thus, we can obtain the finite element implementation of (6) by the same kind of reasoning and vector/matrix representation, leading to the finite state-space form

$$
\dot{\hat{\mathbf{X}}}=\mathbf{L}_{N} \hat{\mathbf{X}}-\mathbf{W}_{g e o}+\mathbf{K}_{X}\left(\mathbf{X}_{a c}-\hat{\mathbf{X}}\right)
$$

where $\mathbf{W}_{\text {geo }}$ represents the approximation of $\nabla V_{\text {geo }}(s)$ together with the Neumann boundary conditions.

In the following, we will check the convergence of such finite-difference approximation schemes of diffusion-based observers as exemplified by (6). Note that it is also possible to study existence and uniqueness properties of observer (6) itself, that can offer useful insights on the behavior of the observer. While addressing these issues is, for the sake of simplicity, out of the scope of this paper, we refer to [38], [56] for interesting examples in this matter.

Once the discrete approximation of the trajectory observer (6) is defined, we can check that this implementation is incrementally stable, i.e. that the final estimate is independent of the initial guess or initial conditions. To check this stability property, we will use contraction analysis [27] on the state-space representations (8) and (10). Roughly speaking, contraction analsysis consists of analyzing the Jacobian of a differential equation to conclude exponential convergence of two different trajectories of the same differential equation, making it independent from the initial conditions. In the example of the discrete heat equation (8), the system is said to be contracting because the matrix can easily be proven, as in [30], to be uniformly negative definite (u.n.d.). Hence we have

$$
\left\|\hat{\mathbf{X}}\left(\hat{\mathbf{X}}_{10}, t\right)-\hat{\mathbf{X}}\left(\hat{\mathbf{X}}_{20}, t\right)\right\| \leq\left\|\hat{\mathbf{X}}_{10}-\hat{\mathbf{X}}_{20}\right\| e^{-\beta\left(t-t_{0}\right)}
$$


which is valid for any couple of initial conditions, $\hat{\mathbf{X}}_{10}$ and $\hat{\mathbf{X}}_{20}$, of $\hat{\mathbf{X}}$, where $-\beta(\beta>0)$ is a constant upper bound on $\mathbf{L}_{D}$.

Proving that the trajectory observer (6)-(10) is contracting is only slightly more involved. Indeed, noting that in (10), $\mathbf{W}_{\text {geo }}$ and $\mathbf{X}_{a c}$ are just inputs to the system, computing the Jacobian of (10) means we can restrict the study to the Jacobian of the Laplace operator (with Neumann boundary conditions) (9) with the Dirac-based observer gain (5). Thus, in order to show that $\mathbf{L}_{N}-\mathbf{K}_{X}$ is uniformly negative definite, one has just to ensure that, in the case where only one acoustic positioning measurement is available, the following matrix is uniformly positive definite.

$$
\left(\begin{array}{ccccccc}
1 & -1 & 0 & 0 & \ddots & \ddots & 0 \\
-1 & 2 & -1 & \ddots & \ddots & \ddots & \ddots \\
0 & -1 & \ddots & \ddots & \ddots & \ddots & \ddots \\
0 & \ddots & \ddots & 2+K & -1 & \ddots & 0 \\
\ddots & \ddots & \ddots & -1 & \ddots & \ddots & 0 \\
\ddots & \ddots & \ddots & \ddots & \ddots & 2 & -1 \\
0 & \ddots & \ddots & 0 & 0 & -1 & 1
\end{array}\right)
$$

Using Sylvester's lemma and computing the principal minors of (12), it can be seen that $\Delta_{i}=1$ for all $i$ until $i=k$ the index of the available acoustic positioning measurement for which $\Delta_{k}=1+K$. For $i>k, \Delta_{i}=1+(i-k+1) K$. The last principal minor $\Delta_{n}$, i.e. the determinant of (12) is then equal to $K$. Thus, all minors $\Delta_{i}$ are positive, hence (12) is uniformly positive definite, meaning that the discrete version of $\nabla^{2} \hat{X}-k_{X}(s) \hat{X}$ is contracting, as well as the implementation of observer (6) with one acoustic positioning measurement.

Using the fact that the sum of two or more contracting systems is also contracting (see the Appendix, Lemma 1), contraction is then proven for the observer for any finite number of acoustic positioning measurements.

Additionally, note that the above observer, and similarly for the other observers described in this paper, can be easily modified using a nonlinear diffusion term as follows

$$
\frac{\partial \hat{X}}{\partial t}=\nabla f\left(\nabla \hat{X}-V_{g e o}\right)+k_{X}\left(X_{a c}-\hat{X}\right)
$$

with only minor modification in the proof of convergence. Indeed, contraction can still be concluded for (13) provided one assumes some mild condition of linear bounded growth on $f$ (see [29]). Indeed, one has

$$
\nabla f\left(\nabla \hat{X}-V_{g e o}\right)=\frac{\partial f}{\partial\left(\nabla \hat{X}-V_{\text {geo }}\right)} \nabla\left(\nabla \hat{X}-V_{g e o}\right)
$$

Such a nonlinear version of the diffusion term could be of interest, for example, in cases where the noise is not Gaussian [14] or to explicitely incorporate outliers into the filtering process (a first attempt has been done in this direction with a nonlinear version of the reaction term $k_{X}\left(X_{a c}-\hat{X}\right)$ in [52]).

Also, one can introduce a more complex gain function $k_{X}(s)$ by replacing (5) with

$$
k_{X}(s)=\sum_{i=1}^{n_{s}} K_{i} \delta\left(s-s_{i}\right)
$$

where the $K_{i}$ are constants that can be tuned independently from each other, thus allowing for more degrees of freedom for the observer.

The reader familiar with image processing techniques has certainly noticed that observers presented in this paper can be seen as being inspired by diffusion-based algorithms that are now well-known and 
pervasive in the literature of the image processing community [43], [55], [21], [14], [38], [48], [28], [53]. Indeed, the vehicle trajectory is in this interpretation just a one-dimensional signal. Though the image signals have generally features that are different from acoustic positioning and doppler signal ones, this perspective definitely gives the advantage of being able to adapt or re-use many interesting methods of diffusion filtering for image processing that lead to significant results.

Observer (13) can also be regarded as a simple continuous realization process. Indeed, using the continuous realization theory terminology [8], [6], [5], this observer is a (gradient) flow, or a deducible procedure used to solve a particular problem, in our case minimizing energy-like integrals such as (4) for instance. As argued by researchers of the continuous realization field, this kind of perspective brings some nice features such as the fact that the algorithm leading to the solution of the problem is contained in one continuous-time problem through the gradient flow (13), but also because its formulation is independent from the chosen method of implementation. Hence, many different implementation methods can be considered.

\section{B. Adding "rendezvous" time constraints}

Some missions of underwater vehicles consist of surveying a sea floor area for cartography and videomosaicking purposes [53]. During these surveys, the path profiles are such that the vehicle happens to go over the same spot at two different times, thus creating loops (see figure 2(a)). When these events are detected for example by cameras, meaning the actual position of the spot is not known, it is of interest to compensate the deviations due to noise on the Doppler sensor by using these "rendezvous" time constraints in the case that only the extremum positions of the considered trajectory are known [11], [10] (see [4]).

The corresponding trajectory observer for a single-loop trajectory can be written as follows.

$$
\begin{aligned}
\frac{\partial \hat{X}}{\partial t}=\nabla(\nabla \hat{X} & \left.-V_{\text {geo }}\right) \\
& +k_{L}\left(\hat{X}\left(s_{2}, t\right)-\hat{X}\left(s_{1}, t\right)\right) \delta\left(s-s_{1}\right) \\
& +k_{L}\left(\hat{X}\left(s_{1}, t\right)-\hat{X}\left(s_{2}, t\right)\right) \delta\left(s-s_{2}\right)
\end{aligned}
$$

in which Dirichlet boundary conditions are assumed. $k_{L}$ is a strictly positive constant, and $s_{1}$ and $s_{2}$ are the two trajectory times for which the positions are the same.

The intuition behind osbserver (14) is quite simple. While the diffusion term is present to give the estimated trajectory the right relative shape, the feedback term favors estimates for which the estimate at time $s_{2}, \hat{X}\left(s_{2}, t\right)$, resembles the estimate at time $s_{1}, \hat{X}\left(s_{1}, t\right)$, and vice-versa. The same reasoning applies to trajectories with several loops. Note the relative simplicity of the method compared to the probabilisticbased approach used in [4].

As for observer (6), checking the contraction property is not difficult if one uses the fact that observer (14) is contracting if the sum of the following matrices is u.n.d.

$$
\left(\begin{array}{ccccc}
-2 & 1 & 0 & 0 & \ddots \\
1 & -2 & 1 & \ddots & \ddots \\
0 & 1 & \ddots & \ddots & 0 \\
0 & \ddots & \ddots & -2 & 1 \\
\ddots & \ddots & 0 & 1 & -2
\end{array}\right)+k_{L}\left(\begin{array}{cccccc}
0 & 0 & \ddots & \ddots & \ddots & 0 \\
0 & -1 & \ddots & \ddots & 1 & \ddots \\
\ddots & \ddots & 0 & \ddots & \ddots & 0 \\
\ddots & \ddots & \ddots & \ddots & \ddots & \ddots \\
\ddots & 1 & \ddots & \ddots & -1 & \ddots \\
0 & \ddots & 0 & \ddots & \ddots & 0
\end{array}\right)
$$

where the first matrix corresponds to the Jacobian approximation of the Laplace operator with Dirichlet conditions, and the second one, which contains the terms induced by the observer feedback, stands for 


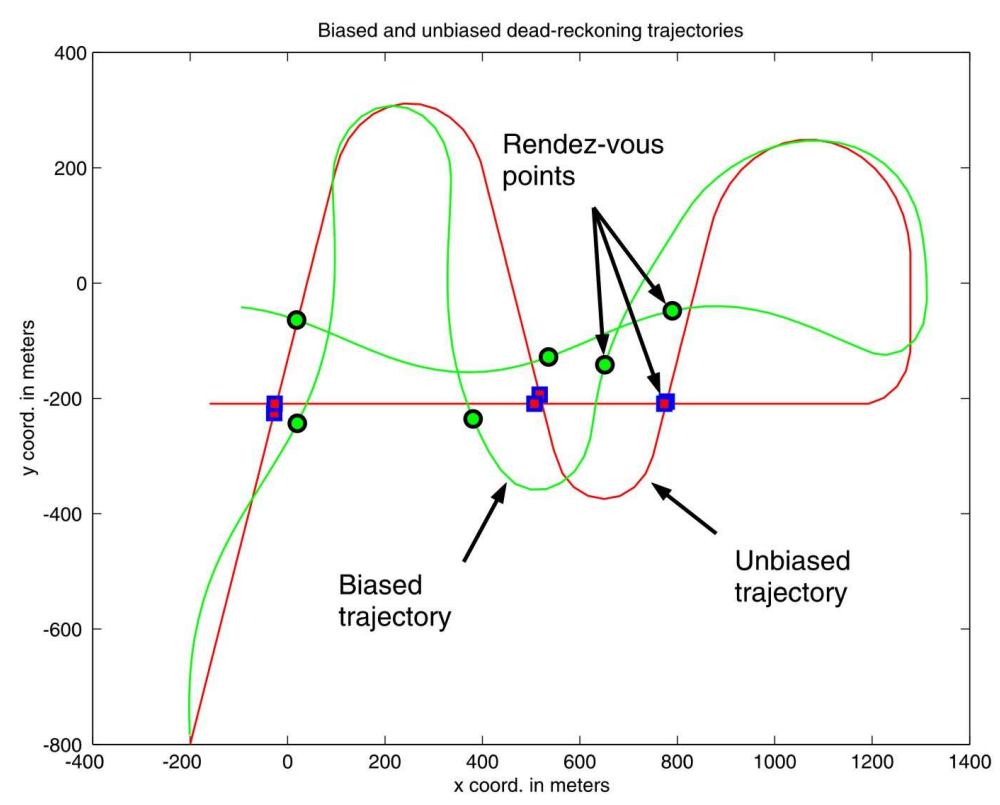

(a) Unbiased and biased trajectory

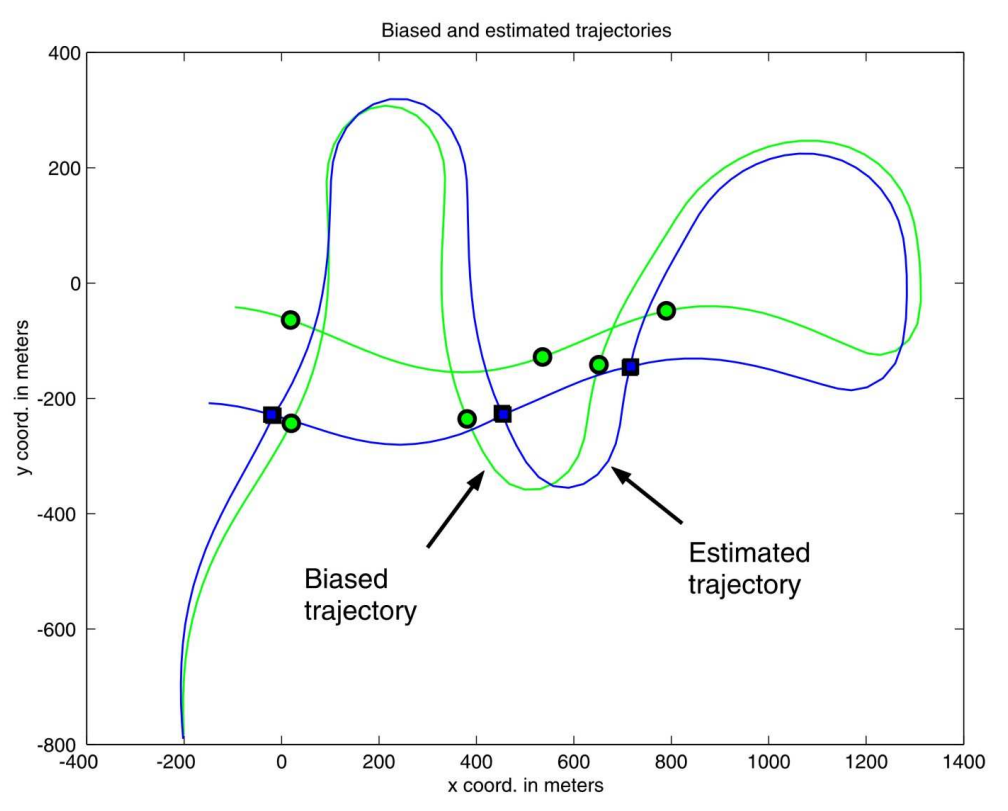

(b) The biased trajectory and its diffusion-based correction

Fig. 2. Simulation of the rendez-vous points problem for a three-loop trajectory

the rendezvous time constraints.

It is straightforward to show that the latter matrix is uniformly negative semi-definite, and that this subsystem is therefore semi-contracting, while the former matrix is u.n.d according to [30]. The contraction property of (14) directly follows from the fact that the sum of a contracting system with a semi-contracting one is contracting (see Appendix, Lemma 1).

Figure 2(a) shows the simulation of a real trajectory (unbiased trajectory) of a vehicle and a Dopplerbased version of it, in which the Doppler sensor was highly corrupted by a low-frequency noise (biased trajectory). The dots indicate the "rendezvous" trajectory times as detected by a camera. It can be seen from the so-called biased trajectory that the error induced by the slow noise affects the rendezvous constraints as each meeting point is quite distant from its counterpart (see the rendezvous points pointed by the arrows). This problem is significantly corrected using a three-loop version of the above observer as shown in figure 2(b) where the rendezvous constraints are recovered in the estimated trajectory.

At this point, one can also draw some interesting connections between our approach and the field of SLAM -Simultaneous Localization And Mapping in which robots use perceptions to build representations of their environment to navigate. Indeed, as in the seminal paper by Lu and Milios [33] -with however different framework/purposes, our approach uses a collection of positions at different time instants, in our case a continuum, to account for the history of the system. Note, interestingly, that in the same work, it is considered that the first position is assumed constant and known, thus playing the role of a Dirichlet boundary condition in a diffusion-based framework. See also the interesting [9], [10] for works on SLAM in an underwater navigation context.

\section{Acoustic data smoothing}

In the case when Doppler measurements are not available, and the objective is to obtain a smooth interpolation $X_{s}$ of the acoustic positioning data, one can use regularization techniques such as Tikhonov regularization [49], [3], [16] which consists, roughly speaking, of requiring that the final estimate as well as its derivatives up to the order $p$ are continuous. 


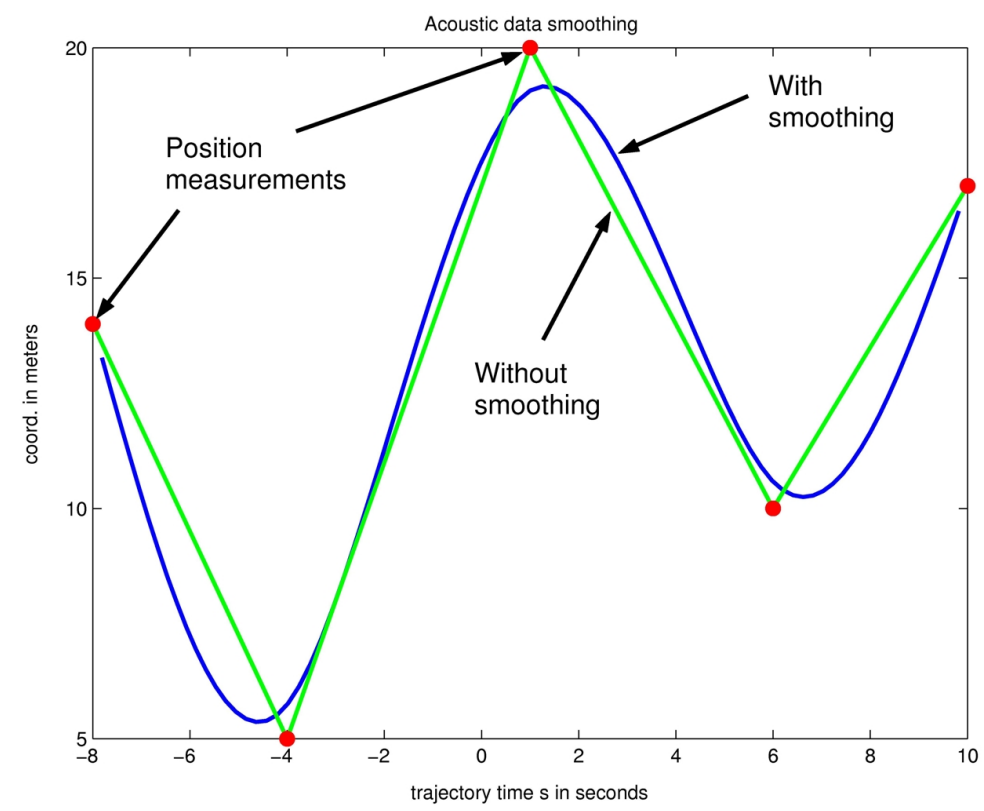

Fig. 3. Simulation of the acoustic data smoothing problem

This constraint can be imposed by using a functional such as the Sobolev norm

$$
\left\|v\left(X_{s}\right)\right\|_{p}^{2}=\int_{s_{b}}^{s_{e}} \sum_{r=0}^{p} q_{r}(s)\left(\nabla^{r} X_{s}\right)^{2} d s
$$

where $q_{r}(s)$ is a pre-specified, non-negative, and continuous weighting function the derivative of each order [48], [49, p. 70].

Hence, restricting the interpolation process to the second order (i.e. $p=2$ ), the integral criterion (16) is now replaced by

$$
J\left(X_{s}\right)=\int_{s_{b}}^{s_{e}}\left\|v\left(X_{s}\right)\right\|_{2}^{2}+k_{X}(s)\left\|X_{a c}-X_{s}\right\|^{2} d s=\int_{s_{b}}^{s_{e}} D\left(\nabla X_{s}\right)^{2}+D_{s}\left(\nabla^{2} X_{s}\right)^{2}+k_{X}(s)\left(X_{s}-X_{a c}\right)^{2} d s
$$

where $D$ and $D_{s}$ are two positive constants. The gradient flow corresponding to this criterion, obtained with the Euler-Lagrange equations, is now

$$
\frac{\partial X_{s}}{\partial t}=D \nabla^{2} X_{s}-D_{s} \nabla^{4} X_{s}+k_{X}(s)\left(X_{a c}-X_{s}\right)
$$

where the conditions at the boundaries are $\hat{X}\left(s_{b}, t\right)=X_{b}, \hat{X}\left(s_{e}, t\right)=X_{e}$ and $\nabla^{2} \hat{X}\left(s_{b}, t\right)=\nabla^{2} \hat{X}\left(s_{e}, t\right)=$ 0 . Contracting behavior of the discrete-space approximation of this PDE can still be concluded by following reasoning similar to that used one in section III-A.

We illustrate this by the simulation of (17) in figure 3, where the effect of the second-order term can be seen through the interpolating effect it creates.

\section{Sensor misalignment and trajectory estimation}

In the case where there is a misalignment in the way the gyrocompass is mounted on the vehicle, there is an undesirable constant bias in the heading measurement (see [22], [23], [35] on the misalignment problem) and the rotation (3) becomes

$$
V_{d}=e^{i \psi_{m}} V_{d o p}
$$


where $\psi_{m} \triangleq \psi+\psi_{b}$ is the angle actually measured because of the constant bias $\psi_{b}$ induced by the misalignment. Combining now (3) with (18), we have the relation between the bias-free velocity vector $V_{\text {geo }}$ and the "disturbed" vector $V_{d}$

$$
V_{g e o}=b V_{d}
$$

where $b \triangleq e^{-i \psi_{b}}$ is the constant unknown imaginary number standing for the rotation due to the gyro misalignment. We would like to have an estimate of this unknown parameter in combination with the diffusion-based estimation process of the previous section.

Introduce the following adaptive-like observer

$$
\frac{\partial \hat{X}}{\partial t}=\nabla\left(\nabla \hat{X}-\hat{b} V_{d}\right)+K\left(X_{s}-\hat{X}\right)
$$

with the adaptation law

$$
\dot{\hat{b}}=-\int_{s_{b}}^{s_{e}} \nabla V_{d}\left(X_{s}-\hat{X}\right) d s
$$

where $\hat{b}(t)$ is the bias estimate, and $X_{s}(s)$ is a smoothed version of the acoustic positioning signal $X_{a c}(s)$. An ODE or space approximation of (20)-(21) is

$$
\begin{aligned}
\dot{\hat{\mathbf{X}}} & =\mathbf{L} \hat{\mathbf{X}}-\mathbf{W}_{d} \hat{b}+K(\mathbf{X}-\hat{\mathbf{X}}) \\
\dot{\hat{b}} & =-\mathbf{W}_{d}^{T}(\mathbf{X}-\hat{\mathbf{X}})
\end{aligned}
$$

where $\hat{\mathbf{X}} \in \mathbb{R}^{n}$ is the finite dimensional state approximation of $\hat{X}(s, t), \mathbf{L}$ a Laplacian matrix and $\mathbf{W}_{d}$ the approximation of $\nabla V_{d}(s)$.

In order to ensure stable behavior of observer (22)-(23), it is required that in the absence of noise and uncertainties, $\hat{\mathbf{X}}$ and $\hat{b}$ converge to the true trajectory and gyro bias

$$
\begin{aligned}
\frac{\partial X}{\partial t} & =\nabla\left(\nabla X-b V_{d}\right) \\
\dot{b} & =0
\end{aligned}
$$

whose approximation is

$$
\begin{aligned}
\dot{\mathbf{X}} & =\mathbf{L X}-\mathbf{W}_{d} b \\
\dot{b} & =0
\end{aligned}
$$

Then, combining (22)-(23) with (24)-(25), we have the following error dynamics

$$
\frac{d}{d t}\left(\begin{array}{c}
\tilde{\mathbf{X}} \\
\tilde{b}
\end{array}\right)=\left(\begin{array}{cc}
\mathbf{L}+K \mathbf{I} & -\mathbf{W}_{d} \\
\mathbf{W}_{d}^{T} & 0
\end{array}\right)\left(\begin{array}{c}
\tilde{\mathbf{X}} \\
\tilde{b}
\end{array}\right)
$$

(where $\tilde{\mathbf{X}}=\hat{\mathbf{X}}-\mathbf{X}$ and $\tilde{b}=\hat{b}-b$ ) which is often encountered in adaptive control [37], [15], [46].

Using the results of Loria and Panteley [31], [32], and under appropriate assumptions on the matrix $\mathbf{W}_{d}$ which are reminiscent of the persistency of excitation condition, one can conclude exponential convergence of (26) and hence of $\hat{\mathbf{X}}$ and $\hat{b}$ towards $\mathbf{X}$ and $b$.

In figure 4(a), we took a trajectory $X(s)$, computed its derivative and created an artificial and exaggerated gyro misalignment of 5 degrees, which, after time integration, gives the rotated trajectory $X_{d}(s)$. The blue line representing $\hat{X}$ shows the behavior of observer (20)-(21) which estimates $X(s)$ properly, while figure 4 (b) represents the evolution of $\hat{b}$ reaching the bias value. 


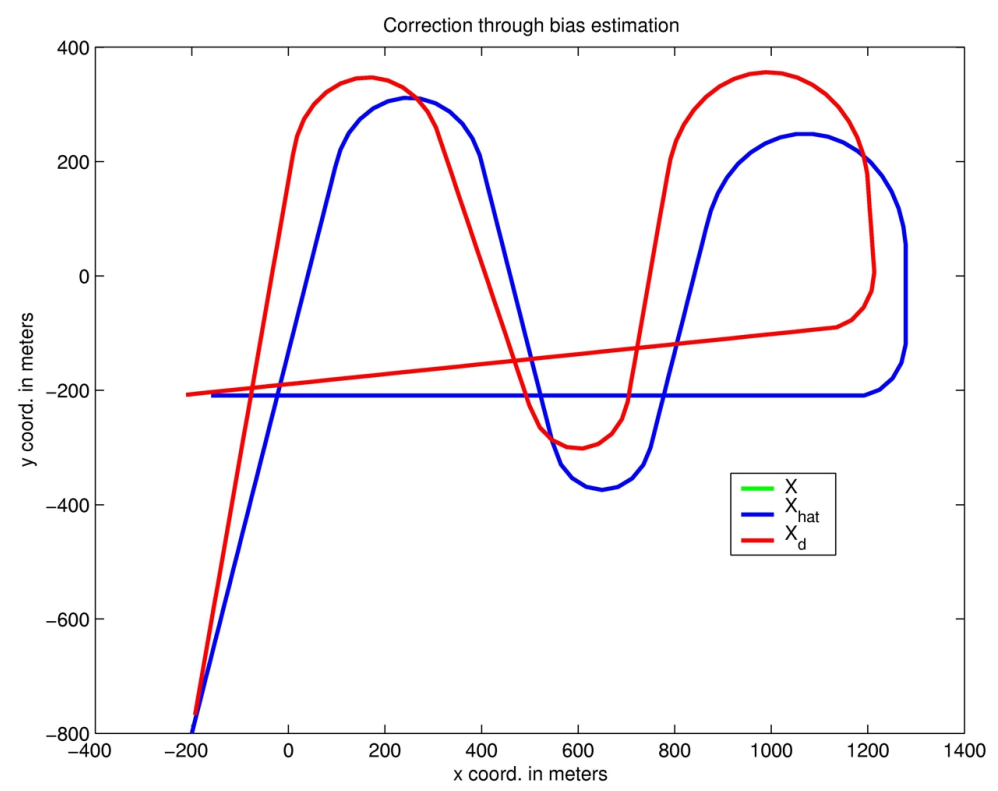

(a) Biased and unbiased trajectories

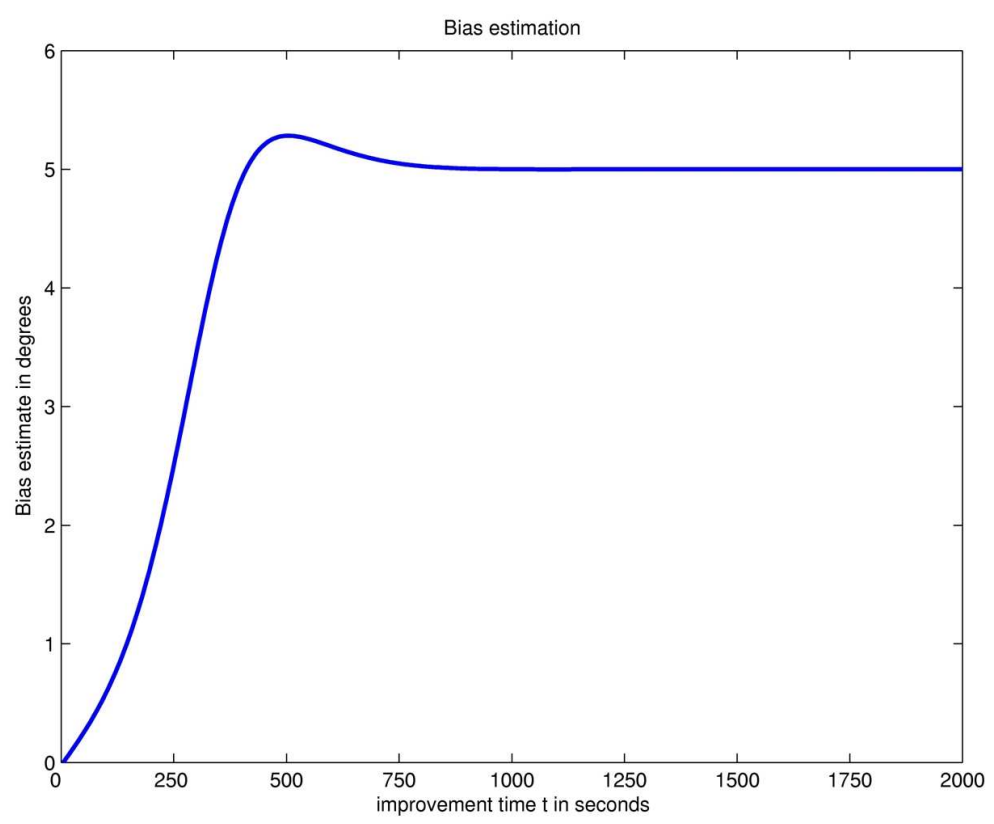

(b) Bias estimate

Fig. 4. Simulation of the sensor misalignment problem

\section{E. Sound velocity profile estimation}

Other problems induced by error in the parameters might occur in the trajectory estimation process (see for example the early work [45] on different error sources in acoustic transponders). Consider for example the simple case depicted in figure 5 where a vehicle moves towards a single transponder that it uses to estimate its longitudinal position knowing the position of the transponder on the sea floor.

In this case, the distance of the vehicle with respect to the transponder, here denoted as $\rho$, is obtained by the relation

$$
\rho=v_{\text {sound }} \frac{T_{\text {tof }}}{2}
$$

where $v_{\text {sound }}$ is the sound velocity and $T_{\text {tof }}$ is the time-of-flight of acoustic pulses measured by the vehicle interrogating the transponder.

It is clear from relation (27) that the distance $\rho$ can be quite inaccurate if the sound velocity at the location of operation is different from the nominal sound velocity parameter on board the vehicle. Indeed, consider that such a difference is expressed by the following expression

$$
\rho_{d}=\frac{v_{\text {sound }}}{a} \frac{T_{\text {tof }}}{2}
$$

where the positive "multiplicative" scalar term a accounts for the mismatch between the real velocity profile $v_{\text {sound }}$ along the trajectory, and $v_{\text {sound }} / a$ that the vehicle uses to compute its position from $T_{\text {tof }}$.

Assuming that we have at our disposal the derivative of the distance, i.e. $\nabla \rho_{\text {geo }}$, obtained thanks to a Doppler sensor, an observer estimating both the distance $\rho$ and the parameter $a$, would take the form

$$
\begin{aligned}
\frac{\partial \hat{\rho}}{\partial t} & =\nabla\left(\nabla \hat{\rho}-\nabla \rho_{g e o}\right)+k_{\rho}\left(\rho_{d} \hat{a}-\hat{\rho}\right) \\
\dot{\hat{a}} & =k_{a}\left(\int_{s_{b}}^{s_{e}} \hat{\rho} d s / \int_{s_{b}}^{s_{e}} \rho_{d} d s-\hat{a}\right)
\end{aligned}
$$

where $k_{a}$ is a scalar gain for the parameter estimator (29). 
Alternatively, if we now consider that parameter $a$ may vary along the trajectory by letting $a=a(s)$, i.e. that we now have a sound velocity profile (see [7] for an interesting study), replace observer (28)-(29) with

$$
\begin{aligned}
& \frac{\partial \hat{\rho}}{\partial t}=\nabla\left(\nabla \hat{\rho}-\nabla \rho_{\text {geo }}\right)+k_{\rho}(s)\left(\rho_{d} \hat{a}(s)-\hat{\rho}\right) \\
& \frac{\partial \hat{a}}{\partial t}=\nabla^{2} \hat{a}+k_{a}(s)\left(\frac{\hat{\rho}}{\rho_{d}}-\hat{a}\right)
\end{aligned}
$$

whose ODE version is

$$
\begin{aligned}
& \dot{\hat{\rho}}=\mathbf{L}_{\rho} \hat{\rho}-\mathbf{W}_{\rho}+\mathbf{K}_{\rho}\left(\operatorname{diag}\left(\rho_{d}\right) \hat{\mathbf{a}}-\hat{\rho}\right) \\
& \dot{\hat{\mathbf{a}}}=\mathbf{L}_{a} \hat{\mathbf{a}}-\mathbf{W}_{a}+\mathbf{K}_{a}\left(\operatorname{diag}^{-1}\left(\rho_{d}\right) \hat{\rho}-\hat{\mathbf{a}}\right)
\end{aligned}
$$

where $\mathbf{L}_{\rho}$ and $\mathbf{L}_{a}$ are Laplacian matrices, $\mathbf{W}_{\rho}$ is a matrix including the boundary conditions and the approximation of $\nabla^{2} \rho_{\text {geo }}$, while $\mathbf{W}_{a}$ contains only the boundary conditions of $\hat{a}$. Then, exponential convergence of (32)-(33) can be guaranteed using the contracting version of the small-gain theorem (see Appendix, Lemma 2) by writing the differential gains of, respectively (32) and (33)

$$
\gamma_{\rho}=\frac{\left\|\mathbf{K}_{\rho}\right\|}{\beta_{\rho}} \max \left(\rho_{d}\right)
$$

where the strictly positive constant $\beta_{\rho}$ is such that $\mathbf{L}_{\rho}-\mathbf{K}_{\rho} \leq-\beta_{\rho} I$, and

$$
\gamma_{a}=\frac{\left\|\mathbf{K}_{a}\right\|}{\beta_{a}} \frac{1}{\min \left(\rho_{d}\right)}
$$

where $\mathbf{L}_{a}-\mathbf{K}_{a} \leq-\beta_{a} I$, thus leading to the condition

$$
\frac{\left\|\mathbf{K}_{\rho}\right\|}{\beta_{\rho}} \frac{\left\|\mathbf{K}_{a}\right\|}{\beta_{a}}<\frac{\min \left(\rho_{d}\right)}{\max \left(\rho_{d}\right)}
$$

that the feedback gains $\mathbf{K}_{\rho}$ and $\mathbf{K}_{a}$ have to verify.

Note that observer (30)-(31) can also include in some sense the scalar parameter estimation of observer (28)-(29). To illustrate this, consider the simulation result shown in figure 6 in which we have artificially induced a multiplicative term $a=0.9$ in the velocity profile. Note that the final estimate $\hat{\rho}$ corresponds to the "true" $\rho$, meaning that $\hat{a}(s)$ has properly estimated the value of the constant parameter $a$ along the trajectory.

\section{F. Online version and further extensions}

The trajectory observers that were previously depicted can be simply extended. For example, if instead of an offline/batch-like trajectory observer (like the one in (6)), a real-time version of a trajectory observer would be

$$
\frac{\partial \hat{X}}{\partial t}=V_{\text {geo }}(s, t)+\nabla\left(\nabla \hat{X}-V_{\text {geo }}(s, t)\right)+k_{X}\left(X_{a c}-\hat{X}\right)
$$

where the term $V_{\text {geo }}(s, t)$ accounts for the fact that for the real trajectory $X(s, t)$, we have $\frac{\partial X}{\partial t}=V_{\text {geo }}(s, t)$, which is the trajectory version of $\dot{X}=V_{\text {geo }}$, and represents the speed at which the trajectory segment to be estimated is moving in time along the whole trajectory (see also [19] for more details).

This observer is still contracting since the additional term $V_{\text {geo }}(s, t)$ is nothing but a time-varying input to the observer.

In figure 7(a) the simulation of such an observer for a one-dimensional trajectory is shown, for which the observer was not initialized on the exact location of the real trajectory. As in a usual Luenberger observer, 


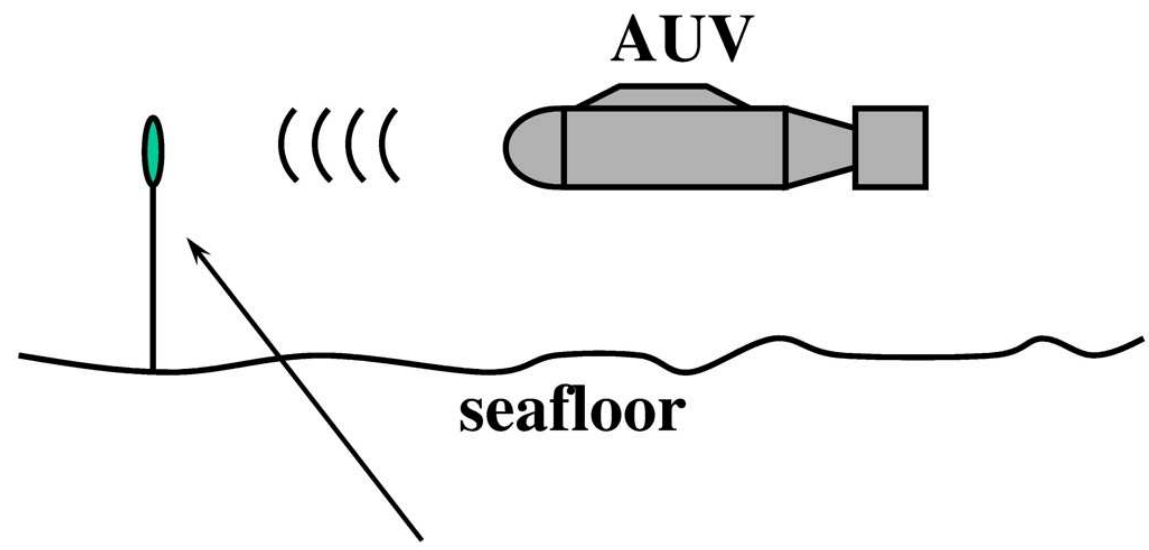

transponder

Fig. 5. Underwater vehicle and acoustic rangemeter

the diffusion-based observer recovers the actual trajectory after a transient. The 3D view of figure 7(b) illustrates the sliding trajectory segment aspect of the same simulation.

Note that observers after eq. (6) can also be implemented as such, i.e. in a more batch-like version where for example the flow (6) can be solved for each instant of time, where the initial condition is chosen as, for example, the estimation of the previous instant, or a shifted version. This point-of-view can also be related to the moving-horizon observer concept (see for example [36]) in the Model Predictive Control literature.

Other simple extensions can also be considered. Indeed, in the case where there are also outages on the Doppler signal, which can happen, for example when the vehicle surveys a zone with a cliff or fault, observer (6) can be augmented and rewritten as

$$
\begin{aligned}
& \frac{\partial \hat{X}}{\partial t}=\nabla(\nabla \hat{X}-\hat{V})+k_{X}\left(X_{a c}-\hat{X}\right) \\
& \frac{\partial \hat{V}}{\partial t}=\nabla^{2} \hat{V}+k_{V}\left(V_{\text {geo }}-\hat{V}\right)
\end{aligned}
$$

where equation (36) takes care of the outages of $V_{\text {geo }}$ in the same way as it is done in (35) for $X_{a c}$.

The overall contracting behavior of observer (35)-(36) is then easily concluded by using the combination result of contracting systems in cascade form (see Appendix, Lemma 3).

Finally, if some acceleration measurements are added to the available information (as provided by an Inertial Measurement Unit for example), equation (36) can be in turn changed into

$$
\frac{\partial \hat{V}}{\partial t}=\nabla\left(\nabla \hat{V}-A_{\text {ins }}\right)+k_{V}\left(V_{\text {geo }}-\hat{V}\right)
$$

which has the same structure as (6) or (35), and for which contracting behavior is concluded identically.

\section{EXPERIMENTAL RESULTS}

Full-scale test trials have been performed at sea during the joint IFREMER-AWI (Alfred Wegener Institut) "Victor in the North" scientific cruise in the Atlantic ocean. IFREMER ROV Victor 6000 was equipped with an Octans II Gyrocompass [39] and an RDI Doppler Velocity Log [54]. Acoustic positioning of the vehicle was performed with the Posidonia long-range USBL system [44], a data being received every 14 seconds. Note that the errors of such a system, if used alone, can be quite significant (see Opderbecke 


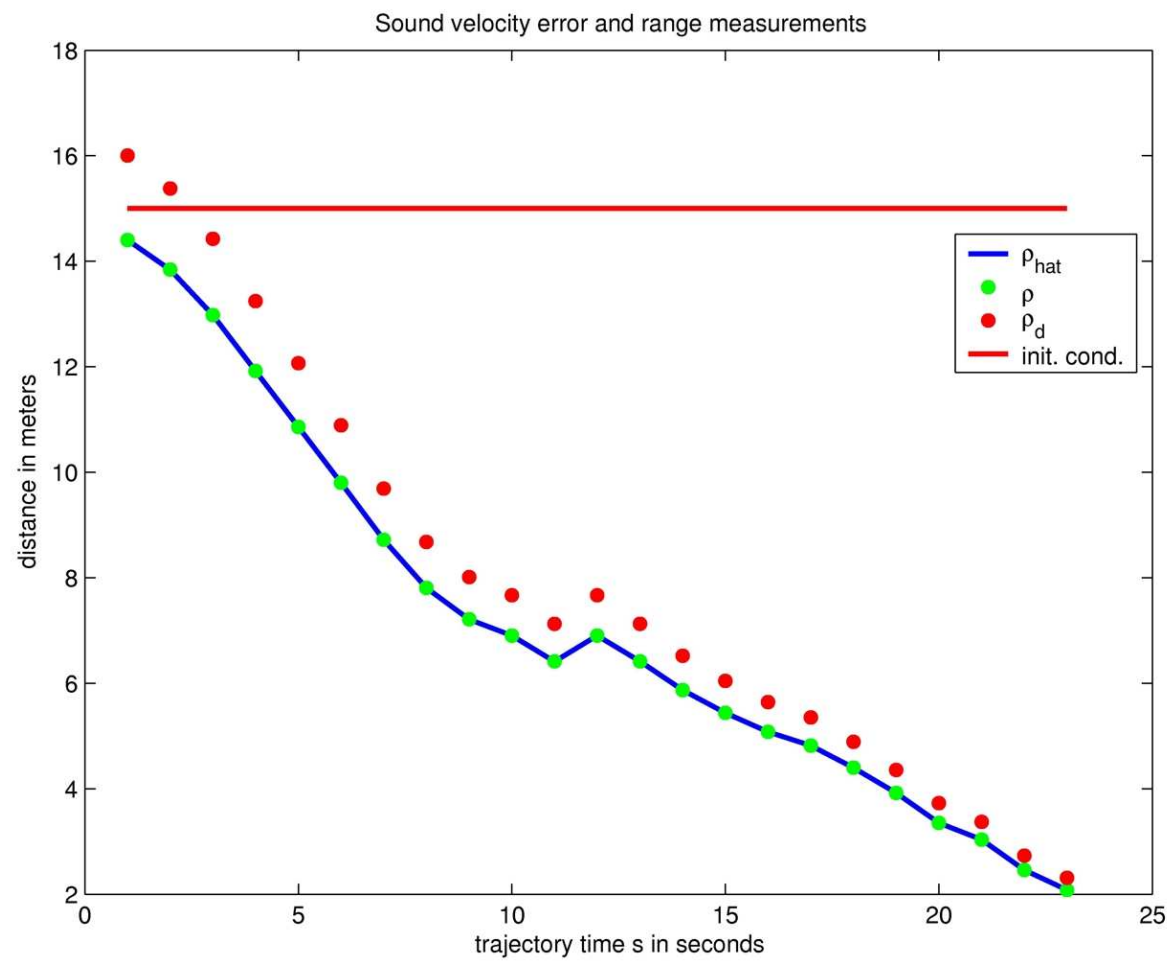

Fig. 6. Sound velocity profile estimation

[42] for a quantitative assessment of the global error of the Posidonia system), and is dependent upon many different factors, hence the process is neither Gaussian nor stationary (see also an interesting discussion on noise in LBL data in [41]).

In figures 8, 9, 10 and 11 a result of the combination of the USBL acoustic positioning system data of Victor 6000 with its gyro-Doppler measurements for a four hour long trajectory is shown, its global view can be seen in figure 8 . Figures 9 and 10 zoom in on some specific parts of the trajectory, specifically the top part and the bottom part of figure 8, with different characteristics. Figure 11 shows a one-dimensional profile to show the behavior of one coordinate with respect to the trajectory time $s$.

The red dots are the acoustic positions delivered by the USBL. As the ROV moves with respect to the vessel, varying noise characteristics affect acoustic positioning. Note the large outliers in the different figures. The starting point of the trajectory (see top of figure 8) defines the initial value to obtain the dead-reckoning trajectory (in green) obtained after integration of gyro-Doppler data. The reader will certainly notice the differences between these two curves. The acoustic positioning-based one has a good global position which, however, is noisy and not smooth, while the gyro-Doppler trajectory behaves nicely regarding the dynamical aspects but is "bent" by error on the integration process.

The blue line in the figures is the result of the estimation process performed by the diffusion-based trajectory observer (6) which was initialized with a crude first-order interpolation of the acoustic positioning data (thin red line). In this experiment, the constant $K$ associated to the feedback gain $k_{X}(s)$ (eq. (5)) is set to $10^{-4}$. Note the good behavior of the observer as it advantageously combines the smooth speed feature of the gyro-Doppler measurements with the absolute positioning of the USBL system. The observer was implemented in Matlab code on a standard PC. Since the gyro-Doppler measurements are obtained at regular sampling intervals, it is quite natural to use finite difference schemes to spatially approximate the PDEs, a consideration which also motivated the difference-scheme based perspective for the stability analysis (see Eq. (9) and onward). For time-discretization, experience has shown that trivial implementation such as the Euler method and higher-order methods could be used, even though 


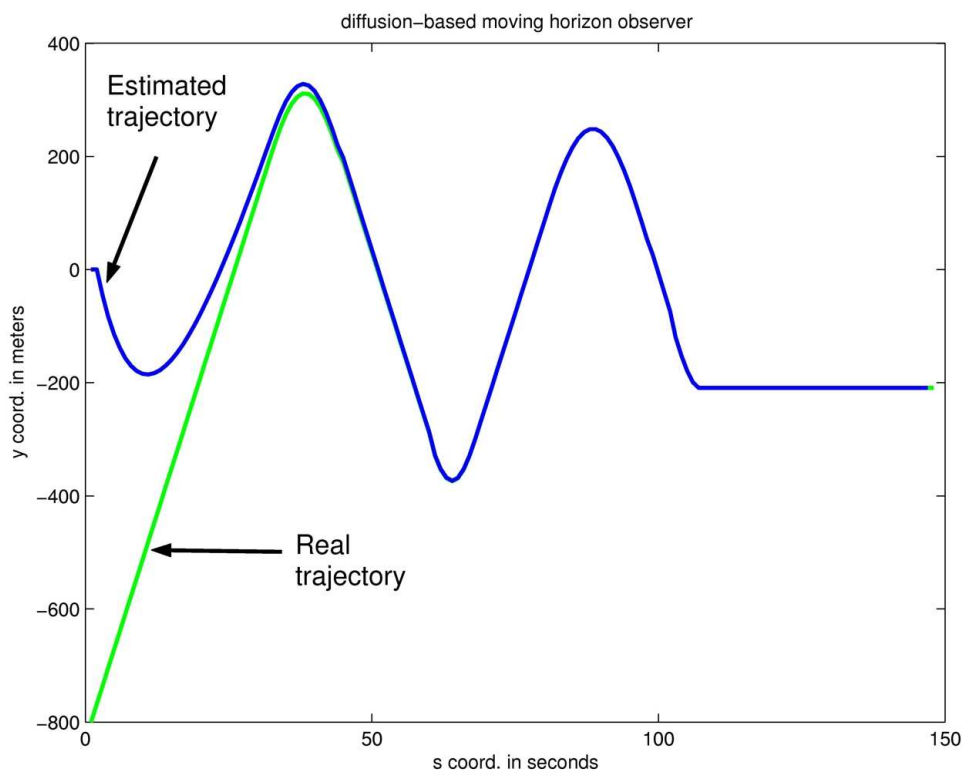

(a) Simulation of observer (34) for a one-dimensional trajectory

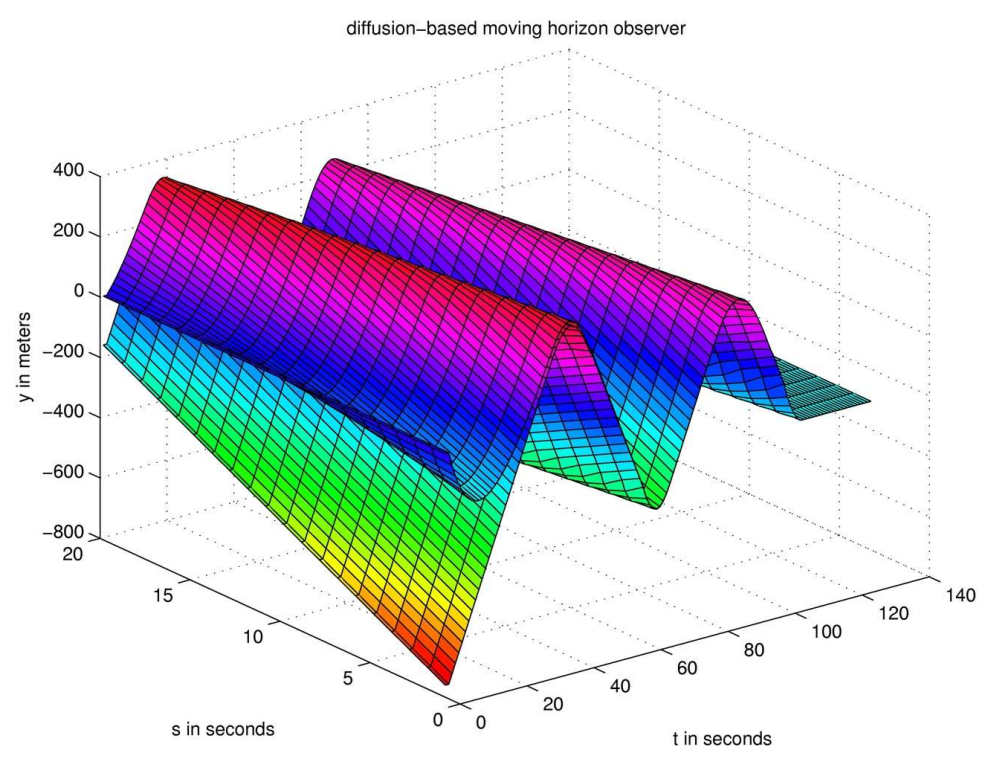

(b) 3D representation of the simulation of observer (34)

Fig. 7. On-line diffusion-based trajectory observer

the sampling period should be small to guarantee good results, a fact that is well-known for diffusionbased equations. To increase performance, we used semi-implicit schemes based on LU decomposition as presented in [56].

On the practical point-of-view, note that these two trajectories are the main information that are available to the operational team and pilots of Victor 6000 to estimate the horizontal location and trajectory of the vehicle. In order to reduce the mismatch between the two trajectories, the initial value of the Dopplerbased trajectory is manually re-initialized every now and then, which creates steps in the trajectory that do not correspond to realistic underwater vehicle dynamics and can therefore be unsuitable for cartography purposes.

\section{CONCLUDING REMARKS}

In this paper, we proposed a simple approach for estimating underwater vehicle trajectories, consisting of processing a whole trajectory segment at a time using a diffusion-based observer, with gyro-Doppler measurements and acoustic positioning signals as inputs. We also presented variations of the same concept to address several important issues related to underwater navigation. Simulated and experimental results were presented to demonstrate the potential of the approach.

Current research includes the introduction of a nonlinear diffusion term, directly deduced from the non-Gaussian, non-stationary nature of the noise of many long-range LBL/USBL positioning systems. Also, it could be of interest to apply the approach to the range-only measurement problem [47], [13], $[50]$.

\section{APPENDIX}

In this appendix, we briefly recall a few elements of contraction analysis (see [27] for the main reference) that are used throughout the paper. In the following, consider systems described by the general nonlinear deterministic differential equation of the form

$$
\dot{\mathbf{x}}=\mathbf{f}(\mathbf{x}, t)
$$




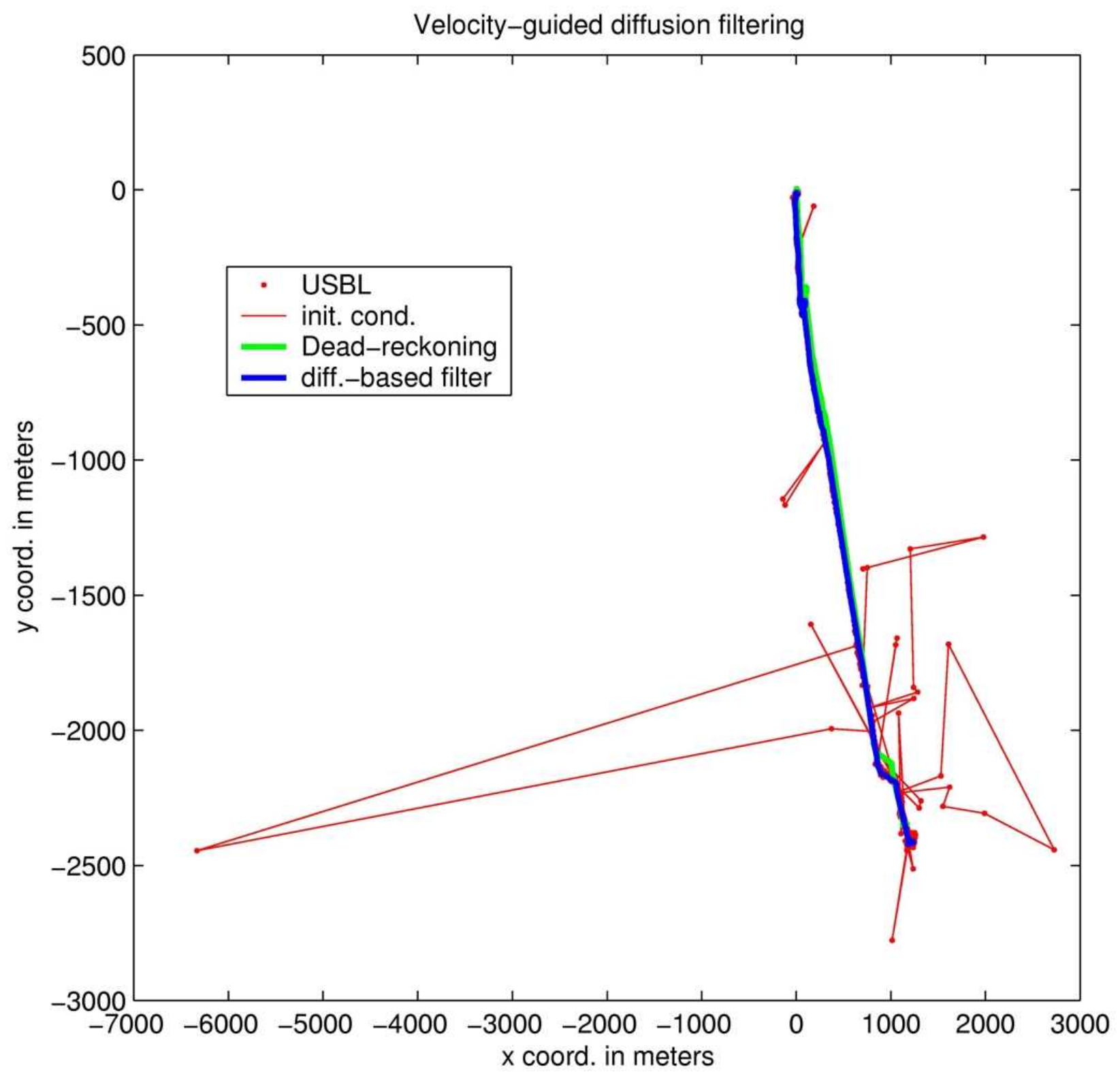

Fig. 8. Experimental result of the diffusion-based trajectory observer (6) (global view of a 4-hour trajectory)

where $\mathbf{x}$ is the $n$-dimensional vector corresponding to the state of the system, and $\mathbf{f}$ is a nonlinear vector field. In addition, we make the further assumption that the system is smooth and that any solution $\mathbf{x}\left(\mathrm{x}_{0}, t\right)$ of (37) exists and is unique.

Let us start by stating the definition of a contracting system.

Definition 1 (contracting system [27]): The system $\dot{\mathbf{x}}=\mathbf{f}(\mathbf{x}, t)$ is said to be contracting if its Jacobian is uniformly negative definite (u.n.d.), i.e. if there exists a strictly positive constant $\beta$, referred to as the contraction rate, such that

$$
\frac{\partial \mathbf{f}}{\partial \mathbf{x}}^{\top}+\frac{\partial \mathbf{f}}{\partial \mathbf{x}} \leq-2 \beta I
$$

for all $\mathbf{x} \in \mathbb{R}^{n}$ and for all $t \geq 0$.

Additionally, the system $\dot{\mathbf{x}}=\mathbf{f}(\mathbf{x}, t)$ is said to be semi-contracting when its Jacobian is only negative semi-definite.

The main theorem of contraction analysis can then be stated as follows. 


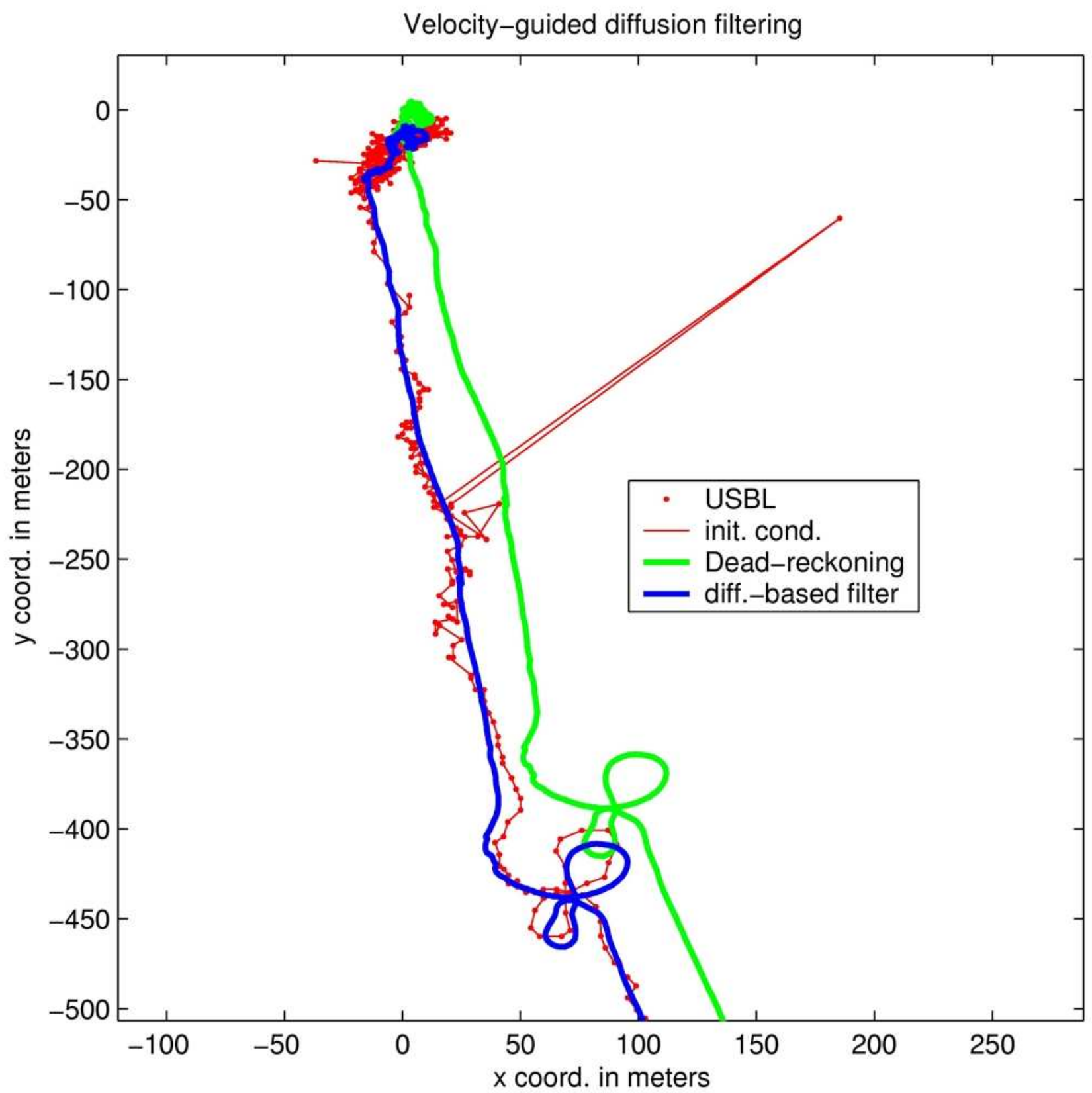

Fig. 9. Experimental result of the diffusion-based trajectory observer (6) (top part)

Theorem 1 (exponential convergence of contracting systems [27]): If the system $\dot{\mathbf{x}}=\mathbf{f}(\mathbf{x}, t)$ is globally contracting with rate $\beta$, then any couple of trajectories $\mathbf{x}_{1}(t)$ and $\mathbf{x}_{2}(t)$ verifies the following inequality

$$
\left\|\mathbf{x}_{1}(t)-\mathbf{x}_{2}(t)\right\| \leq\left\|\mathbf{x}_{10}-\mathbf{x}_{20}\right\| e^{-\beta\left(t-t_{0}\right)}
$$

for all $\mathbf{x}_{10}, \mathbf{x}_{20} \in \mathbb{R}^{n}$ and for all $t_{0} \geq 0, t \geq t_{0}$.

We will now recall some combination properties of contracting systems in the following few lemmas. For the sake of clarity, we have ordered these lemmas according to the order in which they are used in the paper.

Lemma 1 (additive property): Assume that $\dot{\mathbf{x}}=\mathbf{f}(\mathbf{x}, t)$ is contracting and that $\dot{\mathbf{x}}=\mathbf{g}(\mathbf{x}, t)$ is contracting or semi-contracting. Then

$$
\dot{\mathbf{x}}=\mathbf{f}(\mathbf{x}, t)+\mathbf{g}(\mathbf{x}, t)
$$




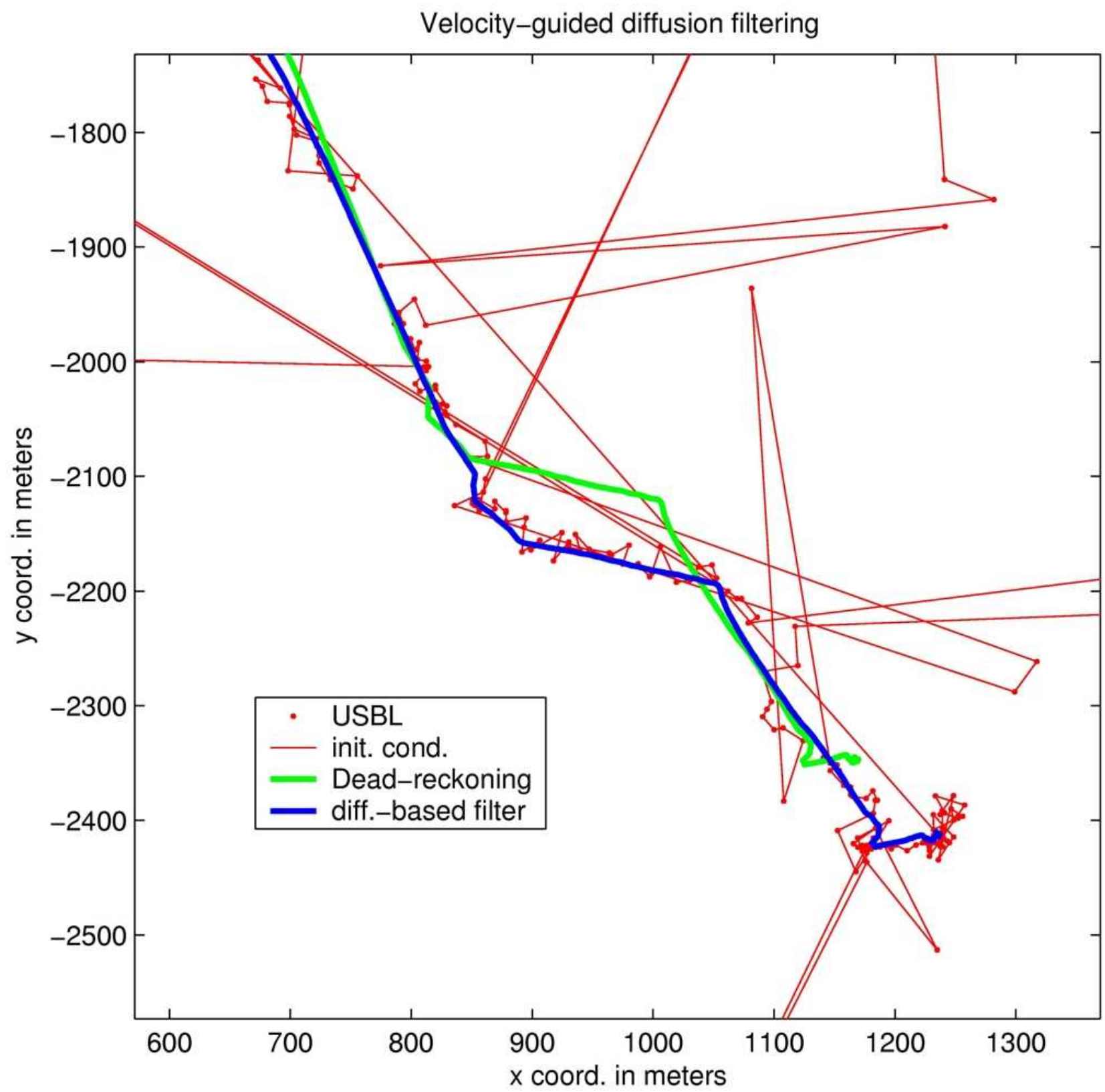

Fig. 10. Experimental result of the velocity-based diffusion-observer (6) (bottom part)

is contracting.

The following definition is quite important for robustness issues and will be of use in the next lemma. Definition 2 ([17]): Consider the following system

$$
\dot{\mathbf{x}}=\mathbf{f}(\mathbf{x}, \mathbf{u}, t)
$$

where $\mathbf{u}$ is an external time-varying signal. Assume that (39) is contracting with rate $\beta$ for all $\mathbf{u}$, and that there exists a positive constant $\sigma$ such that $\frac{\partial \mathbf{f}}{\partial \mathbf{u}}$ is uniformly bounded. Then, the differential gain of (39) is the positive constant $\gamma$ such that

$$
\gamma=\frac{\sigma}{\beta}
$$

Lemma 2 (small-gain theorem [17]): Let two systems be interconnected as follows.

$$
\left\{\begin{array}{c}
\dot{\mathbf{x}}_{1}=\mathbf{f}_{1}\left(\mathbf{x}_{1}, \mathbf{x}_{2}, t\right) \\
\dot{\mathbf{x}}_{2}=\mathbf{f}_{2}\left(\mathbf{x}_{1}, \mathbf{x}_{2}, t\right)
\end{array}\right.
$$




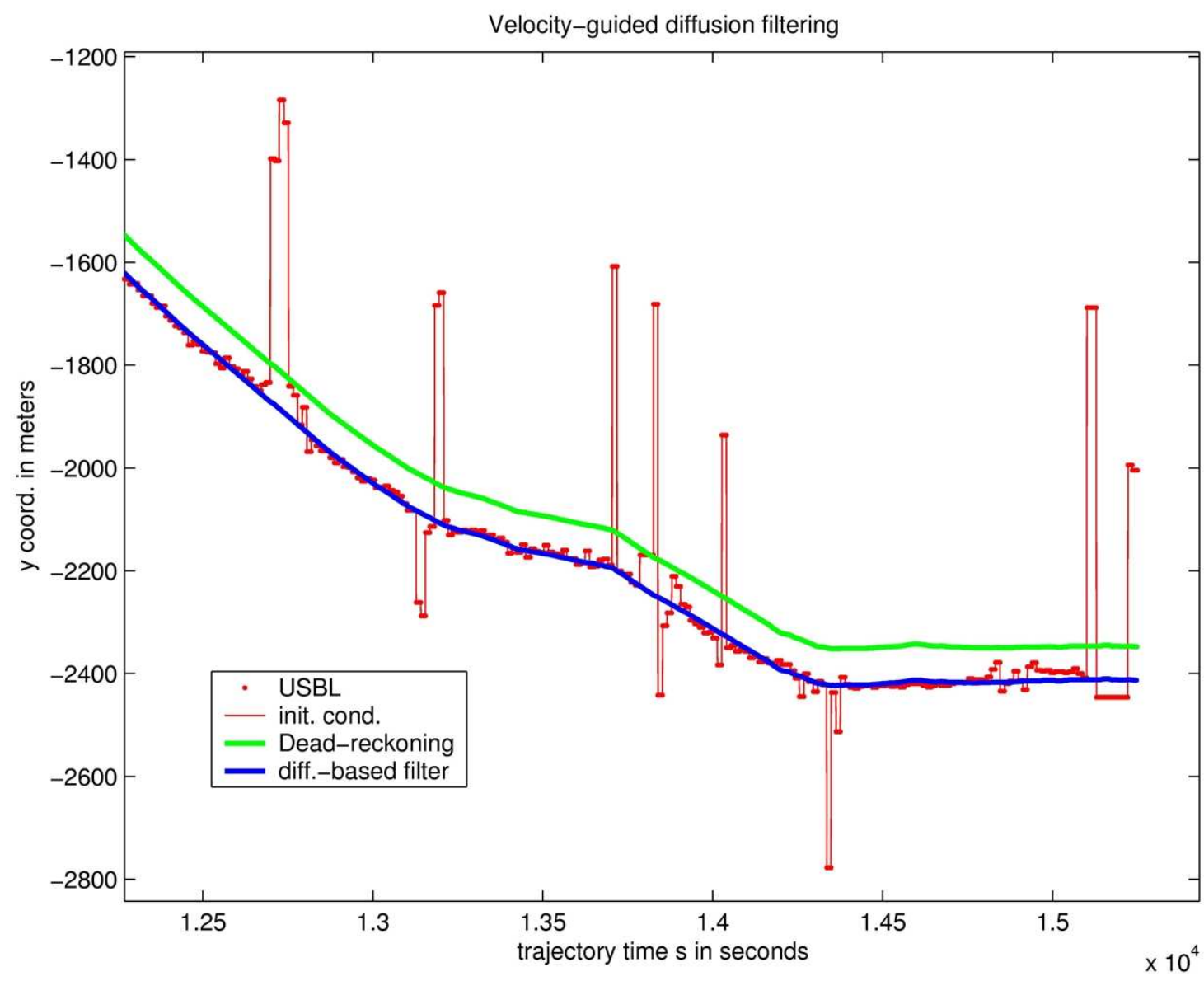

Fig. 11. Experimental result of the velocity-based diffusion-observer (6) (zoom on the y-coordinates)

If $\dot{\mathbf{x}}_{1}$ and $\dot{\mathbf{x}}_{2}$ are contracting, and that their respective differential gains $\gamma_{1}$ and $\gamma_{2}$ are such that

$$
\gamma_{1} \gamma_{2}<1
$$

then the global system (40) is contracting.

Lemma 3 (cascade of two contracting systems[27]): Let two systems be in cascade form as follows.

$$
\left\{\begin{array}{l}
\dot{\mathbf{x}}_{1}=\mathbf{f}_{1}\left(\mathbf{x}_{1}, t\right) \\
\dot{\mathbf{x}}_{2}=\mathbf{f}_{2}\left(\mathbf{x}_{1}, \mathbf{x}_{2}, t\right)
\end{array}\right.
$$

If $\dot{\mathbf{x}}_{1}$ and $\dot{\mathbf{x}}_{2}$ are contracting, and that $\frac{\partial \mathbf{f}_{2}}{\partial \mathbf{x}_{1}}$ is uniformly bounded, then the global system (41) is contracting.

\section{ACKNOWLEDGMENTS}

This work benefited from many interesting discussions with the pilots of Victor 6000. The authors would also like to thank Thor I. Fossen and Andrew Ross, as well as the anonymous reviewers for their many useful remarks and suggestions.

\section{REFERENCES}

[1] A. Alcocer, P. Oliveira, A. Pascoal, "Study and Implementation of an EKF GIB-based underwater positioning system," in Proc. of the IFAC Conference on Control Applications in Marine Systems (CAMS'04), Ancona, Italy, 2004.

[2] P. E. An, A. J. Healey, J. Park, and S. M. Smith, "Asynchronous data fusion for AUV navigation via heuristic fuzzy filtering techniques," in Proc. IEEE Oceans'97, 1997, pp. 397-402.

[3] T. Berg, J. Jouffroy and V. Johansen, "A trajectory observer for camera-based underwater motion measurements," in Proc. IEEE Oceans'04, Kobe, Japan, 2004. 
[4] M. Borgetto, C. Jauffret, and V. Rigaud, "Auto-localisation d'un véhicule sous-marin exploratoire quadrillant une zone," in Proc. GRETSI '03, Paris, France, 2003.

[5] R. W. Brockett, "Least squares matching problems," Linear Algebra Appl., vol. 122-124, pp. 761-777, 1989.

[6] R. W. Brockett, "Dynamical systems that sort lists, diagonalize matrices, and solve linear programming problems," Linear Algebra Appl., vol. 146, pp. 79-91, 1991.

[7] J. V. Candy and E. J. Sullivan, "Sound velocity profile estimation: a system theoretic approach," IEEE Journal of Oceanic Engineering, vol. 18 , no. 3, pp. 240-252, 1993.

[8] M. T. Chu, "A list of matrix flows with applications," Fields Institute Communications, vol. 3, pp. 87-97, 1991.

[9] R. Eustice, O. Pizarro and H. Singh, "Visually augmented navigation in an unstructured environment using a delayed state history," in Proc. IEEE Int. Conf. on Robotics and Automation, New Orleans, LA, 2004.

[10] R. Eustice, H. Singh and J. J. Leonard, "Exactly sparse delayed-state filters," in Proc. IEEE Int. Conf. on Robotics and Automation, Barcelona, Spain, 2005.

[11] S. D. Fleischer, S. M. Rock and R. Burton, "Global position determination and vehicle path estimation from a vision sensor for real-time video mosaicking and navigation,” in Proc. IEEE Oceans'97, Halifax, NS, 1997.

[12] T. I. Fossen, Guidance and control of ocean vehicles. John Wiley \& Sons, 1994.

[13] A. Gadre and D. J. Stilwell, "Toward underwater navigation based on range measurements from a single location," in Proc. IEEE Int. Conf. on Robotics and Automation, New Orleans, LA, 2004.

[14] A. B. Hamza, H. Krim, and G. B. Unal, "Unifying probabilistic and variational estimation," IEEE Signal Processing Magazine, vol. 19, no. 5, pp. 37-47, 2002.

[15] P. Ioannou and J. Sun, Robust Adaptive Control. Prentice-Hall, New Jersey, 1996.

[16] T. Johansen, "On Tikhonov Regularization, Bias and Variance in Nonlinear System Identification," Automatica, vol. 33, no. 3, pp. 441-446, 1997.

[17] J. Jouffroy, "A simple extension of contraction theory to study incremental stability properties," in European Control Conference, Cambridge, UK, 2003.

[18] J. Jouffroy, "A relaxed criterion for contraction theory: application to an underwater vehicle observer," in European Control Conference, Cambridge, UK, 2003.

[19] J. Jouffroy and T. D. Nguyen, "Towards on-line underwater vehicle trajectory estimation using trajectory observers," in Proc. IEEE Oceans'04, Kobe, Japan, 2004.

[20] J. Jouffroy and J. Opderbecke, "Underwater vehicle trajectory estimation using contracting PDE-based observers," in American Control Conference, Boston, MA, 2004.

[21] M. Kass, A. Witkin and D. Terzopoulos, "Snakes: active contour models," Int. Journal of Computer Vision, vol. 1, no. 4, pp. 321-331, 1987.

[22] J. C. Kinsey and L. L. Whitcomb, "Towards in-situ calibration of gyro and doppler navigation sensors for precision underwater vehicle navigation," in Proc. IEEE Int. Conf. on Robotics and Automation, Washington, DC, 2002.

[23] J. C. Kinsey and L. L. Whitcomb, "Adaptive identification on the group of rigid body rotations" in IEEE Int. Conf. on Robotics and Automation, Barcelona, Spain, 2005.

[24] D. Kristiansen, "Modeling of cylinder gyroscopes and observer design for nonlinear oscillations," Ph.D. dissertation, Dep. Eng. Cybernetics, NTNU, Trondheim, Norway, 2000.

[25] C. Lanczos, The variational principles of mechanics (4th ed.). Dover, New York, 1970.

[26] W. Lohmiller and J.-J. E. Slotine, "On metric observers for nonlinear observers" in IEEE Int. Conf. on Control Applications, Dearborn, Michigan, 1996.

[27] W. Lohmiller and J.-J. E. Slotine, "On contraction analysis for non-linear systems," Automatica, vol. 34, no. 6, pp. 683-696, 1998.

[28] W. Lohmiller and J.-J. E. Slotine, "Global convergence rates of nonlinear diffusion for time-varying images," in $2 n d$ Int. Conf. on Scale-Space Theories in Computer Vision, Corfu, Greece, 1999.

[29] W. Lohmiller and J.-J. E. Slotine, "On stability of nonlinear reaction-diffusion processes," in European Control Conference, Karlsruhe, Germany, 1999.

[30] W. Lohmiller and J.-J. E. Slotine, "Stability analysis and observer design for nonlinear diffusion processes," in New trends in nonlinear observer design, Nijmeijer. H. and Fossen. T. I., Eds. Springer-Verlag, 1999, pp. 93-111.

[31] A. Loria and E. Panteley, "Uniform exponential stability of linear time-varying systems: revisited," Systems and Control Letters, vol. 47, no. 1, pp. 13-24, 2002.

[32] A. Loria, "Explicit convergence rates for MRAC-type systems," Automatica, vol. 40, pp. 1465-1468, 2004.

[33] F. Lu and E. Milios, "Globally consistent range scan alignment for environment mapping," Autonomous Robots, vol. 4, no. 4, pp. 333-349, 1997.

[34] D. G. Luenberger, "An introduction to observers," IEEE Transactions on Automatic Control, vol. AC-16, no. 6, pp. 596-602, 1971.

[35] R. McEwen, H. Thomas, D. Weber and F. Psota, "Performance of an AUV navigation system at arctic latitures," IEEE Journal of Oceanic Engineering, vol. 30, no. 2, pp. 443-454, 2005.

[36] H. Michalska and D. Q. Mayne, "Moving horizon-observers and observer-based control," IEEE Transactions on Automatic Contol, vol. 40, no. 6, pp. 995-1006, 1995.

[37] K. S. Narendra and A. M. Annaswamy, Stable Adaptive Systems. Prentice-Hall, New Jersey, 1989.

[38] K. N. Nordström, "Biased anisotropic diffusion: a unified regularization and diffusion approach to edge detection," Image and vision computing, vol. 8, no. 4, pp. 318-327, 1990.

[39] Octans III Gyrocompass and motion sensor, Ixsea, http://www.ixsea.com/downloads/sales_documentation/uk_Octans_v0503.pdf

[40] P. Oliveira and A. Pascoal, "Navigation systems design: an application of multi-rate filtering," in Proc. IEEE Oceans'98, pp. 1348-1353, 1998.

[41] E. Olson, J. Leonard and S. Teller, "Robust range-only beacon localization,” in Proc. IEEE AUV'04, 2004. 
[42] J. Opderbecke, "Précision théorique du positionnement en mode reconnaissance," Internal Report IFREMER, La-Seyne-Sur-Mer, France, 1999.

[43] P. Perona and J. Malik, "Scale-space and edge detection using anisotropic diffusion," IEEE Transactions on Pattern Analysis and Machine Intelligence, vol. 12, no. 7, pp. 629-639, 1990.

[44] Posidonia Long Range USBL, Ixsea, http://www.ixsea.com/downloads/sales_documentation/uk_Posidonia_v0403.pdf

[45] J. R. Short and S. P. Travis, "A real time transponder location method," in Proc. IEEE Oceans'73 Conference, pp. 583-590, 1973.

[46] J.-J. E. Slotine and W. Li, Applied Nonlinear Control. Prentice-Hall, New Jersey, 1991.

[47] T. L. Song, "Observability of target tracking with range-only measurements," IEEE Journal of Oceanic Engineering, vol. 24, no. 3, pp. 383-387, 1999.

[48] D. Terzopoulos, "Regularization of inverse visual problems involving discontinuities," IEEE Transactions on pattern analysis and Machine Intelligence, vol. 8, no. 4, pp. 413-424, 1986.

[49] A. N. Tikhonov and V. Y. Arsenin, Solutions of Ill-Posed Problems, Scripta series in Mathematics. V.H. Winston \& Sons, Washington, D.C., english translation edition, 1977.

[50] J. Vaganay, P. Baccou and B. Jouvencel, “Homing by acoustic ranging to a single beacon,” in Proc. IEEE Oceans'O0, Providence, RI, 2000.

[51] B. Vik and T. I. Fossen, "A nonlinear observer for GPS and INS integration," in Proc. IEEE Conf. on Decision and Control, Orlando, Florida USA, 2001.

[52] S. Vike and J. Jouffroy, "Diffusion-based outlier rejection for underwater navigation," in Proc. IEEE Oceans'05, Washington, D.C., 2005.

[53] A. G. Vincent, N. Pessel, M. Borgetto, J. Jouffroy, J. Opderbecke, and V. Rigaud, "Real-time geo-referenced video mosaicking with the MATISSE system," in Proc. IEEE Oceans'03, San Diego, CA, 2003.

[54] Workhorse Navigator Doppler Velocity Log (DVL), RD Instruments, http://www.rdinstruments.com/pdfs/datasheets/workhorse_nav_ds_lr.pdf

[55] J. Weickert, Anisotropic diffusion in image processing. Teubner, Stuttgart, 1998.

[56] J. Weickert, B. M. ter Haar Romeny, and M. A. Viergever, "Efficient and reliable schemes for nonlinear diffusion filtering," IEEE Transactions on Image Processing, vol. 7, no. 3, pp. 398-410, 1998.

[57] L. L. Whitcomb, D. R. Yoerger, and H. Singh, "Advances in Doppler-based navigation of underwater robotic vehicles," in Proc. Int. Conf. on Robotics and Automation, Detroit, Michigan, 1999.

[58] L. L. Whitcomb, D. R. Yoerger, and H. Singh, "Combined Doppler / LBL based navigation of underwater vehicles," in Proc. Int. Symposium on Unmanned Untethered Submersible Technology, Durham, New Hampshire, 1999. 